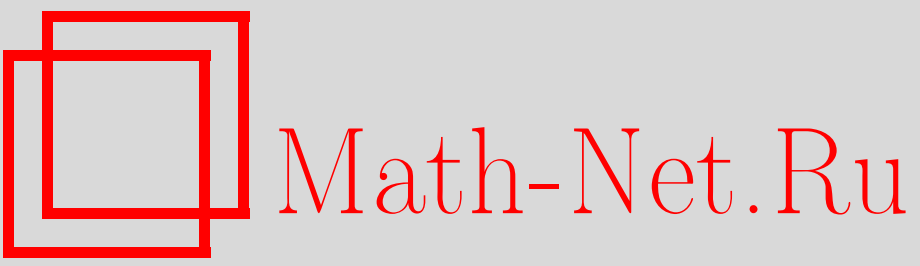

В. М. Адуков, О факторизации аналитических матриц-функций, ТМФ, 1999, том 118, номер 3, 324-336

DOI: https://doi.org/10.4213/tmf704

Использование Общероссийского математического портала Math-Net.Ru подразумевает, что вы прочитали и согласны с пользовательским соглашением

http://www . mathnet.ru/rus/agreement

Параметры загрузки:

IP : 54.237 .206 .68

26 апреля 2023 г., $17: 45: 25$ 
ТЕОРЕТИЧЕСКАЯ

И МАТЕМАТИЧЕСКАЯ

ФИЗИКА

Том 118, № 3

март, 1999

(C) 1999 г.

\author{
В.М. Адуков*
}

\title{
О ФАКТОРИЗАЦИИ АНАЛИТИЧЕСКИХ МАТРИЦ-ФУНКЦИЙ
}

Предложен новый метод явного вычисления частных индексов и построения факторизации Винера-Хопфа аналитических матриц-функций, имеющий вычислительные преимущества по сравнению с ранее развитым методом автора. Предложенный метод применяется для нахождения делителей аналитической матрицы-функции, ответственных за нули ее определителя.

\section{1. ВВЕДЕНИЕ}

Краевая задача Римана для вектора, детально изученная в связи с исследованием систем сингулярных интегральных уравнений, в последние годы стала привычным аппаратом математической физики. Введение ее в обиход этой теории связано в основном с двумя важными работами: статьей [1], где сушественно использовалась краевая задача Римана при интегрировании нелинейных эволюционных уравнений методом обратной задачи рассеяния, и статьей [2], в которой краевая задача Римана была положена в основу решения обратной задачи рассеяния для матричного дифференциального оператора.

Исследование задачи Римана в настоящее время принято проводить с помощю решения следующей факторизационной задачи.

Пусть $\Gamma$ - простой гладкий замкнутый контур в комплексной плоскости $\mathbb{C}$, ограничиваюший область $D_{+}$. Дополнение $D_{+} \cup \Gamma$ в расширенной комплексной плоскости $\mathbb{C} \cup\{\infty\}$ обозначим через $D_{-}$. Можно считать, что $0 \in D_{+}, \infty \in D_{-}$. Пусть $a(t)$ - непрерывная и обратимая на контуре Г матрица-функция порядка $p$.

Правой факторизачией Винера-Хопфа матрицы-функции $a(t)$ называется ее представление в виде

$$
a(t)=r_{-}(t) d_{r}(t) r_{+}(t), \quad t \in \Gamma,
$$

где $r_{ \pm}(t)$ - непрерьвные на Г матрицы-функции, аналитически продолжимые в область $D_{ \pm}$и обратимые там, $d_{r}(t)=\operatorname{diag}\left[t^{\rho_{1}}, \ldots, t^{\rho_{p}}\right]$. Здесь $\rho_{1}, \ldots, \rho_{p}$ - целые числа,

* Южно-Уральский государственный университет, Челябинск, Россия. E-mail: adukov@math.tu-chel.ac.ru 
называемые правыми частными индексами $a(t)$, причем их можно упорядочить по возрастанию: $\rho_{1} \leqslant \cdots \leqslant \rho_{p}$.

Аналогично определяется левая факторизация $a(t)$ :

$$
a(t)=l_{+}(t) d_{l}(t) l_{-}(t), \quad t \in \Gamma,
$$

$d_{l}(t)=\operatorname{diag}\left[t^{\lambda_{1}}, \ldots, t^{\lambda_{p}}\right], \lambda_{1} \geqslant \cdots \geqslant \lambda_{p},-$ левъе частные индексы $a(t)$. Легко видеть, что

$$
\sum_{j=1}^{p} \rho_{j}=\sum_{j=1}^{p} \lambda_{j}=\kappa,
$$

где $\kappa=\operatorname{ind}_{\Gamma} \operatorname{det} a(t)$ - индекс Коши определителя $a(t)$.

Известно, что факторизация Винера-Хопфа существует для матриц-функций с гельдеровскими на контуре Г элементами либо с элементами из алгебры Винера. Однако явных формул для факторизационных множителей и частных индексов в общем случае нет, что сушественно сдерживает дальнейшие применения краевой задачи Римана.

Поэтому основной задачей в этой области является поиск случаев явного решения задачи факторизации. Один из таких случаев (мероморфной в области $D_{+}$матрицы-функции) был установлен в работе [3]. Если ограничиться для простоты аналитическими матрицами-функциями, то оказывается, что явное решение задачи факторизации требует использования $2 \kappa+1$ моментов функции $a^{-1}(t)$ относительно контура $\Gamma$.

В этой работе предлагается метод, основанный на тех же идеях, что и в работе [3], но более простой с вычислительной точки зрения, поскольку он использует $2 \kappa+1$ моментов более простой матрицы-функции $\Delta_{-}^{-1}(t) a(t)$. Здесь $\Delta_{-}^{-1}(t)-$ скалярный многочлен от $t^{-1}$, являюшийся множителем в факторизации Винера-Хопфа $\Delta(t)=$ $\Delta_{-}(t) t^{-\kappa} \Delta_{+}(t)$ определителя $\Delta(t)=\operatorname{det} a(t)$.

\section{2. ИНДЕКСЫ И СУЩЕСТВЕННЫЕ МНОГОЧЛЕНЫ КОНЕЧНОЙ ПОСЛЕДОВАТЕЛЬНОСТИ МАТРИЦ}

В этом разделе развивается техника, необходимая для получения в явном виде факторизации Винера-Хопфа. Все построения носят чисто алгебраический характер. Доказательства утверждений этого раздела опушены, их можно найти в статьях $[3,4]$.

Преж де всего введем понятия индексов и существенных многочленов конечной последовательности матриц.

Обозначим через $\mathbb{C}^{p \times q}$ множество всех комплексных матрищ размера $p \times q$. Для матрицы $A$ мы будем обозначать через $\operatorname{ker}_{R} A$ ее правое ядро и $\operatorname{ker}_{L} A$ левое ядро:

$$
\operatorname{ker}_{R} A=\{x \mid A x=0\}, \quad \operatorname{ker}_{L} A=\{y \mid y A=0\} .
$$

Пусть $A$ - блочная матрица с блоками из $\mathbb{C}^{p \times p}$, имеюшая блочные размеры $(n+1) \times$ $(m+1)$. Разобъем строку $L \in \operatorname{ker}_{L} A$ на $n+1$ блоков:

$$
L=\left(l_{0} l_{-1} \ldots l_{-n}\right),
$$


и определим для $L$ производящий векторный многочлен от переменной $t^{-1}$ :

$$
L(t)=l_{0}+l_{-1} t^{-1}+\cdots+l_{-n} t^{-n} .
$$

Аналогично для столбца из правого ядра $\operatorname{ker}_{R} A$ определим производяший векторный многочлен от переменной $t$.

Пусть $c_{-m}, \ldots, c_{0}, \ldots, c_{n}$ - конечная последовательность комплексных матриц порядка $p$. Образуем семейство блочных теплицевых матриц

$$
T_{k}=\left\|c_{i-j}\right\|_{\substack{i=k, k+1, \ldots, n \\ j=0,1, \ldots, m+k}}
$$

$(-m \leqslant k \leqslant n)$. Для того чтобы ввести понятия индексов и существенных многочленов последовательности, изучим структуру правого и левого ядер матрицы $T_{k}$.

Поскольку удобнее иметь дело не с векторами, а с производяшими векторными многочленами, перейдем от пространств $\operatorname{ker}_{R} T_{k}$ и $\operatorname{ker}_{L} T_{k}$ к изоморфным пространствам $\mathcal{N}_{k}^{R}$ и $\mathcal{N}_{k}^{L}$ производящих векторных многочленов. Чтобы сделать это, введем операторы $\sigma_{R}$ и $\sigma_{L}$. Для $p=1$ оператор $\sigma_{R}=\sigma_{L}$ является функционалом Стилтьеса, используемым в теории ортогональных многочленов.

На пространстве рациональных матриц-функций вида

$$
R(t)=\sum_{j=-n}^{m} r_{j} t^{j}, \quad r_{j} \in \mathbb{C}^{p \times l},
$$

определим оператор

$$
\sigma_{R}(R(t))=\sum_{j=-n}^{m} \mathrm{c}_{-j} r_{j} .
$$

Мы используем обозначение $\sigma_{R}$ для всех $l \geqslant 1$, поскольку это не вызывает недоразумений.

Через $\mathcal{N}_{k}^{R}(-m \leqslant k \leqslant n)$ обозначим пространство векторных многочленов вида

$$
R(t)=\sum_{j=0}^{m+k} r_{j} t^{j}, \quad r_{j} \in \mathbb{C}^{p \times 1},
$$

таких, что

$$
\sigma_{R}\left(t^{-i} R(t)\right)=0, \quad i=k, k+1, \ldots, n .
$$

Легко видеть, что $\mathcal{N}_{k}^{R}$ - пространство производящих многочленов векторов из $\operatorname{ker}_{R} T_{k}$. Для удобства положим $\mathcal{N}_{-m-1}^{R}=0$ и обозначим $(n+m+2) p$-мерноепространство всех столбцовых многочленов формальной степени $n+m+1$ через $\mathcal{N}_{n+1}^{R}$. 
Аналогично на пространстве матриц-функций вида

$$
L(t)=\sum_{j=-n}^{m} l_{j} t^{j}, \quad l_{j} \in \mathbb{C}^{l \times p}
$$

определим оператор $\sigma_{L}$ в пространство $\mathbb{C}^{l \times p}$ :

$$
\sigma_{L}\{L(t)\}=\sum_{j=-n}^{m} l_{j} c_{-j} .
$$

Пространство $\operatorname{ker}_{L} T_{k}$ естественно изоморфно пространству $\mathcal{N}_{k}^{L}$ векторных многочленов от $t^{-1}$ вида

$$
L(t)=\sum_{j=0}^{n-k} l_{j} t^{-j}, \quad l_{j} \in \mathbb{C}^{1 \times p}
$$

таких, что

$$
\sigma_{L}\left\{t^{-i} L(t)\right\}=0, \quad i=k, k-1, \ldots,-m .
$$

Положим $\mathcal{N}_{n+1}^{L}=0$ и обозначим $(n+m+2) p$-мерное пространство всех строчных многочленов от $t^{-1}$ формальной степени $n+m+1$ через $\mathcal{N}_{-m-1}^{L}$.

ОПРЕДЕЛЕНИЕ 2.1. Если $\operatorname{dim} \mathcal{N}_{-m}^{R}=\operatorname{dim} \mathcal{N}_{n}^{L}=0, \quad$ то последовательность $c_{-m}, \ldots, c_{0}, \ldots, c_{n}$ будем назьвать регулярной.

Через $d_{k}^{R}\left(d_{k}^{L}\right)$ обозначим размерность пространства $\mathcal{N}_{k}^{R}\left(\mathcal{N}_{k}^{L}\right)$. Пусть $\Delta_{k}^{R}=$ $d_{k}^{R}-d_{k-1}^{R}(-m \leqslant k \leqslant n+1)$ и $\Delta_{k}^{L}=d_{k}^{L}-d_{k+1}^{L}(-m-1 \leqslant k \leqslant n)$. Используя формулу Грассмана, легко доказать, что для любой регулярной последовательности матриц справедливы неравенства

$$
\begin{aligned}
0 & =\Delta_{-m}^{R} \leqslant \Delta_{-m+1}^{R} \leqslant \cdots \leqslant \Delta_{n}^{R} \leqslant \Delta_{n+1}^{R}=2 p \\
2 p & =\Delta_{-m-1}^{L} \geqslant \Delta_{-m}^{L} \geqslant \cdots \geqslant \Delta_{n-1}^{L} \geqslant \Delta_{n}^{L}=0 .
\end{aligned}
$$

Из неравенств (3) следует, что сушествуют $2 p$ целых чисел $\mu_{1} \leqslant \cdots \leqslant \mu_{2 p}$ таких, что

$$
\begin{aligned}
& \Delta_{-m}^{R}=\cdots=\Delta_{\mu_{1}}^{R}=0, \\
& \Delta_{\mu_{i}+1}^{R}=\cdots=\Delta_{\mu_{i+1}}^{R}=i, \\
& \Delta_{\mu_{2 p}+1}^{R}=\cdots=\Delta_{n+1}^{R}=2 p .
\end{aligned}
$$

Если $i$-я строка в этих соотношениях отсутствует, считаем, что $\mu_{i}=\mu_{i+1}$. 
ОПРЕДЕЛЕНИЕ 2.2. Целые числа $\mu_{1}, \ldots, \mu_{2 p}$, определенные соотношениями (5), будем называть индексами последовательности $c_{-m}, \ldots, c_{0}, \ldots, c_{n}$.

Нетрудно показать, что

$$
\sum_{j=1}^{2 p} \mu_{j}=-\operatorname{ind} T_{0}=(n-m) p .
$$

Из определения пространств $\mathcal{N}_{k}^{R}$ легко видеть, что $\mathcal{N}_{k}^{R}$ и $t \mathcal{N}_{k}^{R}$ - подпространства $\mathcal{N}_{k+1}^{R}$, причем размерность $h_{k+1}^{R}$ дополнения $H_{k+1}^{R}$ суммы $\mathcal{N}_{k}^{R}+t \mathcal{N}_{k}^{R}$ до всего пространства $\mathcal{N}_{k+1}^{R}$ равна $\Delta_{k+1}^{R}-\Delta_{k}^{R}$. Отсюда и из соотношений (5) следует, что $h_{k+1}^{R} \neq 0$, только если $k=\mu_{i}(i=1, \ldots, 2 p)$. В этом случае $h_{k+1}^{R}$ совпадает с кратностью $\nu_{i}$ индекса $\mu_{i}$. Поэтому для $k \neq \mu_{i}$ имеем

$$
\mathcal{N}_{k+1}^{R}=\mathcal{N}_{k}^{R}+t \mathcal{N}_{k}^{R}
$$

а для $k=\mu_{i}-$

$$
\mathcal{N}_{k+1}^{R}=\mathcal{N}_{k}^{R}+t \mathcal{N}_{k}^{R}+H_{k+1}^{R}
$$

ОПРЕДЕЛЕНИЕ 2.3. Любые многочлены $R_{i}(t), \ldots, R_{i+\nu_{i}-1}(t)$, образующие базис какого-либо дополнения $H_{\mu_{i}+1}^{R}$, будем называть правыми сушественными многочленами последовательности, соответствующими индексу $\mu_{i}$.

Таким образом, для любой регулярной последовательности мы определили $2 p$ правых сушественных многочленов $R_{1}(t), \ldots, R_{2 p}(t)$.

Аналогично для $k \neq \mu_{i}$ имеем

$$
\mathcal{N}_{k-1}^{L}=\mathcal{N}_{k}^{L}+t^{-1} \mathcal{N}_{k}^{L}
$$

а для $k=\mu_{i}-$

$$
\mathcal{N}_{k-1}^{L}=\mathcal{N}_{k}^{L}+t^{-1} \mathcal{N}_{k}^{L}+H_{k-1}^{L}
$$

Выбирая базисы для пространств $H_{\mu_{i}-1}^{L}(1 \leqslant i \leqslant 2 p)$, мы получаем последовательность векторных многочленов $L_{1}(t), \ldots, L_{2 p}(t)$, которые будем называть левъми $с y$ щественными многочленами последовательности $c_{-m}, \ldots, c_{0}, \ldots, c_{n}$.

Оказывется, что векторные многочлены

$$
\left\{R_{j}(t), t R_{j}(t), \ldots, t^{k-\mu_{j}-1} R_{j}(t)\right\}_{j=1,2, \ldots, i}
$$

являются производящими многочленами элементов базиса пространства $\operatorname{ker}_{R} T_{k}$ для $k \in\left(\mu_{i} ; \mu_{i+1}\right], 1 \leqslant i \leqslant 2 p$. Здесь мы положили $\mu_{2 p+1}=n$.

Аналогично векторные многочлены

$$
\left\{L_{j}(t), t^{-1} L_{j}(t), \ldots, t^{-\left(\mu_{j}-k-1\right)} L_{j}(t)\right\}_{j=i, i+1, \ldots, 2 p}
$$


являются производящими многочленами элементов базиса пространства $\operatorname{ker}_{L} T_{k}$ для $k \in\left[\mu_{i-1}, \mu_{i}\right), 1 \leqslant i \leqslant 2 p, \mu_{0}=-m$.

В дальнейшем нам потребуется критерий, который позволял бы проверять, что заданные числа являются индексами, а заданные векторные многочлены суть существенные многочлены данной последовательности матриц. Такой критерий основан на проверке обратимости некоторых тестовых матриц.

Пусть $c_{-m}, \ldots, c_{0}, \ldots, c_{n}$ - регулярная последовательность. Пусть $k_{1}, \ldots, k_{2 p}$ - целые числа такие, что $-m-1 \leqslant k_{1} \leqslant \cdots \leqslant k_{2 p} \leqslant n+1$ и

$$
\sum_{j=1}^{2 p} k_{j}=-\operatorname{ind} T_{0}
$$

Пусть $U_{1}(t), \ldots, U_{2 p}(t) ; \quad V_{1}(t), \ldots, V_{2 p}(t)$ - такие векторные многочлены, что $U_{j}(t) \in N_{k_{j}+1}^{R}$ и $V_{j}(t) \in N_{k_{j}-1}^{L}, 1 \leqslant j \leqslant 2 p$.

Числа $k_{1}, \ldots, k_{2 p}$ являются индексами, а многочлены $U_{1}(t), \ldots, U_{2 p}(t)$ - правыми сушественными многочленами последовательности $c_{-m}, \ldots, c_{0}, \ldots, c_{n}$ тогда и только тогда, когда обратима матрица

$$
\Lambda_{R}=\left(\begin{array}{ccc}
\tilde{\sigma}_{R}\left\{t^{-n-1} U_{1}(t)\right\} & \cdots & \tilde{\sigma}_{R}\left\{t^{-n-1} U_{2 p}(t)\right\} \\
U_{1,0} & \cdots & U_{2 p, 0}
\end{array}\right)
$$

Аналогично $k_{1}, \ldots, k_{2 p}$ - индексы, а $V_{1}(t), \ldots, V_{2 p}(t)$ - левые сушественные многочлены последовательности тогда и только тогда, когда обратима матрица

$$
\Lambda_{L}=\left(\begin{array}{cc}
V_{1, n-\kappa_{1}+1} & \tilde{\sigma}_{L}\left\{t^{-\kappa_{1}} V_{1}(t)\right\} \\
\vdots & \vdots \\
V_{2 p, n-\kappa_{2 p}+1} & \tilde{\sigma}_{L}\left\{t^{-\kappa_{2 p}} V_{2 p}(t)\right\}
\end{array}\right)
$$

Здесь $\tilde{\sigma}_{R}, \tilde{\sigma}_{L}$ - операторы Стилтьеса для последовательности $c_{-m}, \ldots, c_{0}, \ldots, c_{n}$, продолженной вправо произвольной матрицей $c_{n+1} ; U_{j, 0}-$ старший коэффициент многочлена $U_{j}(t)$ и $V_{j, n-\kappa_{j}+1}-$ свободньй член многочлена $V_{j}(t)$.

\section{3. ФАКТОРИЗАЦИЯ АНАЛИТИЧЕСКИХ МАТРИЦ-ФУНКЦИЙ}

Пусть $a(t)$ - матрица-функция, непрерывная и обратимая на контуре Г и аналитически продолжимая в область $D_{+}$. Обозначим $\Delta(t)=\operatorname{det} a(t)$, и пусть

$$
\Delta(t)=\Delta_{-}(t) t^{\kappa} \Delta_{+}(t)
$$

- факторизация Винера-Хопфа $\Delta(t)$. Поскольку $b(t)=t^{\kappa} \Delta_{-}(t) a^{-1}(t)$ - аналитическая в $D_{+}$матрица-функция, то, применив к ней лемму 1.1 статьи [3], легко получить следующие утверждения: 
1) левые $\lambda_{1}, \ldots, \lambda_{p}$ и правые $\rho_{1}, \ldots, \rho_{p}$ частные индексы аналитической матрицыфункции $a(t)$ неотрицательны;

$2)$ строка $\Delta_{-}(t)\left[r_{-}^{-1}(t)\right]_{j}$ есть векторный многочлен от $t^{-1}$ степени не выше $\kappa-\rho_{j}$;

$3)$ столбец $\Delta_{-}(t)\left[l_{-}^{-1}(t)\right]^{j}$ есть векторный многочлен от $t^{-1}$ степени не вьше $\kappa-\lambda_{j}$.

Здесь и далее для матрицы $A$ мы обозначаем через $[A]_{j}$ ее $j$-ю строку, а через $[A]^{j}-j$-й столбец.

Рассмотрим симметричную последовательность $c_{-\kappa}, \ldots, c_{0}, \ldots, c_{\kappa}$, составленную из моментов матрищы-функции $\Delta_{-}^{-1}(t) a(t)$ относительно контура $\Gamma$ :

$$
c_{j}=\frac{1}{2 \pi i} \int_{\Gamma} t^{-j-1} \Delta_{-}^{-1}(t) a(t) d t, \quad j=-\kappa, \ldots, 0, \ldots, \kappa
$$

При продолжении этой последовательности вправо условимся брать в качестве $c_{\kappa+1}$ соответствующий момент указанной матрицы-функции.

Легко видеть, что операторы $\sigma_{R}$ и $\sigma_{L}$ в этом случае действуют по формулам

$$
\begin{gathered}
\sigma_{R}\{R(t)\}=\frac{1}{2 \pi i} \int_{\Gamma} t^{-1} \Delta_{-}^{-1}(t) a(t) R(t) d t \\
\sigma_{L}\{L(t)\}=\frac{1}{2 \pi i} \int_{\Gamma} t^{-1} \Delta_{-}^{-1}(t) L(t) a(t) d t .
\end{gathered}
$$

Введем следующие обозначения:

$$
L_{p+j}(t)=\Delta_{-}(t)\left[r_{-}^{-1}(t)\right]_{j}, \quad R_{j}(t)=t^{\kappa-\lambda_{j}+1} \Delta_{-}(t)\left[l_{-}^{-1}(t)\right]^{j}, \quad j=1, \ldots, p
$$

ПРЕДЛОЖЕНИЕ 3.1. Имеют место соотношения

$$
\begin{aligned}
L_{p+j}(t) & \in \mathcal{N}_{\rho_{j}-1}^{L}, \\
\sigma_{L}\left\{t^{-\rho_{j}} L_{p+j}(t)\right\} & =\left[r_{+}(0)\right]_{j}, \quad L_{p+j, \kappa-\lambda_{j}+1}=0, \quad j=1, \ldots, p ; \\
R_{j}(t) & \in \mathcal{N}_{-\lambda_{j}+1}^{R}, \\
\sigma_{R}\left\{t^{-\kappa-1} R_{j}(t)\right\} & =\left[l_{+}(0)\right]^{j}, \quad R_{j, 0}=0, \quad j=1, \ldots, p .
\end{aligned}
$$

ДокАЗАТЕЛЬСТво. Из левой факторизации матрицы $a(t)$ следует, что

$$
L_{p+j}(t) \Delta_{-}^{-1}(t) a(t)=t^{\rho_{j}}\left[r_{+}(t)\right]_{j}
$$

Поэтому

$$
\sigma_{L}\left\{t^{-l} L_{p+j}(t)\right\}=\frac{1}{2 \pi i} \int_{\Gamma} t^{\rho_{j}-l-1}\left[r_{+}(t)\right]_{j} d t=0
$$


для $l=\rho_{j}-1, \rho_{j}-2, \ldots,-\kappa$, а при $l=\rho_{j}$ получаем $\sigma_{L}\left\{t^{-\rho_{j}} L_{p+j}(t)\right\}=\left[r_{+}(0)\right]_{j}$. Поскольку степень векторного многочлена не больше $\kappa-\rho_{j}$, то $L_{p+j}(t) \in \mathcal{N}_{\rho_{j}-1}^{L}$ и старший коэффишент $L_{p+j, \kappa-\rho_{j}+1}$ этого многочлена равен нулю. Подобным же образом доказывается и вторая часть предложения.

Аналогичное утверждение имеет место и для подходяшим образом исправленных многочленов Тейлора для векторных функций $\left[r_{+}^{-1}(t)\right]^{j},\left[l_{+}^{-1}(t)\right]_{j}$. Это исправление производится следующим образом. Разложим аналитическую в $D_{+}$векторную функцию $\left[r_{+}^{-1}(t)\right]^{j}$ в ряд Тейлора в окрестности $t=0$ и представим ее в виде

$$
\left[r_{+}^{-1}(t)\right]^{j}=\left[\left(r_{+}^{-1}(t)\right)_{\kappa+\rho_{j}+1}\right]^{j}+t^{\kappa+\rho_{j}+2} \phi_{+}^{j}(t) .
$$

Здесь $\left[\left(r_{+}^{-1}(t)\right)_{\kappa+\rho_{j}+1}\right]^{j}-$ многочлен Тейлора степени $\kappa+\rho_{j}+1$ для $\left[r_{+}^{-1}(t)\right]^{j}, \mathrm{a} \phi_{+}^{j}(t)-$ векторная функшия, аналитическая в $D_{+}$и непрерывная в $D_{+} \cup \Gamma$. Введем векторную функцию

$$
\Phi^{j}(t)=\frac{1}{2 \pi i} \int_{\Gamma} t^{\kappa+\rho_{j}+2} \frac{\Delta_{-}(\tau) l_{-}^{-1}(\tau)-\Delta_{-}(t) l_{-}^{-1}(t)}{(\tau-t) \Delta_{-}(\tau)} l_{-}(\tau) \phi_{+}^{j}(\tau) d \tau
$$

Легко видеть, что $\Phi^{j}(t)$ является векторным многочленом от $t$ степени не больше $\kappa+\rho_{j}+1$, у которого отличны от нуля разве лишь последние $\kappa$ коэффициентов. Исправление $\left[\left(r_{+}^{-1}(t)\right)_{\kappa+\rho_{j}+1}\right]^{j}$ произведем с помошью $\Phi^{j}(t)$, определив векторньй многочлен

$$
R_{p+j}(t)=\left[\left(r_{+}^{-1}(t)\right)_{\kappa+\rho_{j}+1}\right]^{j}+\Phi^{j}(t), \quad j=1, \ldots, p,
$$

формальной степени $\kappa+\rho_{j}+1$.

Представив подобным образом $\left[l_{+}^{-1}(t)\right]_{j}$ :

$$
\left[l_{+}^{-1}(t)\right]_{j}=\left[\left(l_{+}^{-1}(t)\right)_{\kappa+\lambda_{j}+1}\right]_{j}+t^{\kappa+\lambda_{j}+2} \psi_{+}^{j}(t),
$$

и определив векторный многочлен от $t$ :

$$
\Psi^{j}(t)=\frac{1}{2 \pi i} \int_{\Gamma} t^{\kappa+\lambda_{j}+2} \psi_{j}^{+}(\tau) r_{-}(\tau) \frac{\Delta_{-}(\tau) r_{-}^{-1}(\tau)-\Delta_{-}(t) r_{-}^{-1}(t)}{(\tau-t) \Delta_{-}(\tau)} d \tau,
$$

исправим многочлен Тейлора $\left[\left(l_{+}^{-1}(t)\right)_{\kappa+\lambda_{j}+1}\right]_{j}$ и определим векторный многочлен от $t^{-1}$

$$
L_{j}(t)=t^{-\left(\kappa+\lambda_{j}+1\right)}\left(\left[\left(l_{+}^{-1}(t)\right)_{\kappa+\lambda_{j}+1}\right]_{j}+\Psi_{j}(t)\right), \quad j=1, \ldots, p,
$$

степени не выше $\kappa+\lambda_{j}+1$. 
ПРЕДЛОЖЕНИЕ 3.2. Имеют место соотношения

$$
\begin{aligned}
R_{p+j}(t) & \in \mathcal{N}_{\rho_{j}+1}^{R}, \\
\sigma_{R}\left\{t^{-\kappa-1} R_{p+j}(t)\right\} & =0, \quad R_{p+j, 0}=\left[r_{+}^{-1}(0)\right]^{j}, \quad j=1, \ldots, p ; \\
L_{j}(t) & \in \mathcal{N}_{-\lambda_{j}-1}^{R}, \\
\sigma_{L}\left\{t^{\lambda_{j}} L_{j}(t)\right\} & =0, \quad L_{j, \kappa+\lambda_{j}+1}=\left[l_{+}^{-1}(0)\right]_{j}, \quad j=1, \ldots, p .
\end{aligned}
$$

ДокАЗАТЕЛЬСТВо этого предложения может быть проведено по схеме доказательства теоремы 1.2 статьи [3] и потому опушено.

Теперь можно связать частные индексы $\lambda_{1}, \ldots, \lambda_{p}, \rho_{1}, \ldots, \rho_{p}$ и факторизационные множители $l_{ \pm}(t), r_{ \pm}(t)$ аналитической матрицы-функции $a(t)$ с индексами и существенными многочленами последовательности $c_{-}, \ldots, c_{0}, \ldots, c_{\kappa}$. Отметим, что одновременно используется и правая, и левая факторизации Винера-Хопфа функции $a(t)$.

ТЕОРема 3.1. Последовательность $c_{-\kappa}, \ldots, c_{0}, \ldots, c_{\kappa}$ является регулярной последовательностью, индексь которой совпадают с числами $-\lambda_{1}, \ldots,-\lambda_{p}$, $\rho_{1}, \ldots, \rho_{p}$, а многочлень $L_{1}(t), \ldots, L_{2 p}(t), \quad R_{1}(t), \ldots, R_{2 p}(t)$ - соответственно с ее левымм и правыми существенными многочленами.

ДокАЗАТЕльство. Докажем прежде всего регулярность последовательности $c_{-\kappa}, \ldots, c_{0}, \ldots, c_{\kappa}$. Легко видеть, что $\Delta_{-}(t) l_{-}^{-1}(t) d_{l}^{-1}(t)$ есть матричный многочлен от $t^{-1}$ степени не выше $\kappa$. Пусть $\Delta_{-}(t) l_{-}^{-1}(t) d_{l}^{-1}(t)=\alpha_{0}+\alpha_{-1} t^{-1}+\cdots+\alpha_{-\kappa} t^{-\kappa}$, тогда

$$
\sigma_{R}\left\{\Delta_{-}(t) l_{-}^{-1}(t) d_{l}^{-1}(t)\right\}=c_{0} \alpha_{0}+c_{1} \alpha_{-1}+\cdots+c_{\kappa} \alpha_{-\kappa}
$$

С другой стороны, учитывая левую факторизацию $a(t)$, имеем

$$
\sigma_{R}\left\{\Delta_{-}(t) l_{-}^{-1}(t) d_{l}^{-1}(t)\right\}=\frac{1}{2 \pi i} \int_{\Gamma} t^{-1} l_{+}(t) d t=l_{+}(0)
$$

поэтому

$$
\alpha_{0}+\alpha_{-1} t^{-1}+\cdots+\alpha_{-\kappa} t^{-\kappa}=l_{+}(0)
$$

Пусть $x \in \operatorname{ker}_{\mathrm{L}} T_{\kappa}$, т.е. $x c_{-\kappa}=\cdots=x c_{\kappa}=0$. Умножая уравнение (6) слева на $x$, получаем $x l_{+}(0)=0$, т.е. $x=0$. Итак, $\operatorname{ker}_{\mathrm{L}} T_{\kappa}=0$ и $\omega=0$. Аналогично доказывается регулярность последовательности слева.

Обозначим $k_{j}=-\lambda_{j}, k_{p+j}=\rho_{j}, j=1, \ldots, p$. В силу выбранной упорядоченности частных индексов имеем $k_{1} \leqslant \cdots \leqslant k_{2 p}$. Кроме того,

$$
\sum_{j=1}^{2 p} k_{j}=0=-\operatorname{ind} T_{0}
$$


Так как последовательность регулярна, то мы можем применить критерий сушественности. Составив тестовые матрицы

$$
\Lambda_{L}=\left(\begin{array}{cc}
l_{+}^{-1}(0) & 0 \\
0 & r_{+}(0)
\end{array}\right), \quad \Lambda_{R}=\left(\begin{array}{cc}
l_{+}(0) & 0 \\
0 & r_{+}^{-1}(0)
\end{array}\right)
$$

и убедившись в их обратимости, получаем утверждение теоремы.

Сушественные многочлены, построенные в предыдущей теореме, обладают дополнительным свойством, важным для дальнейшего.

ОПРЕДЕЛЕНИЕ 3.1. Существенные многочлены $R_{1}(t), \ldots, R_{p}(t), L_{p+1}(t), \ldots, L_{2 p}(t)$ такие, что: 1$)$ свободные члены многочленов $R_{1}(t), \ldots, R_{p}(t)$ равны нулю; 2$)$ старшие коэффициенты многочленов $L_{p+1}(t), \ldots, L_{2 p}(t)$ равны нулю, будем называть факторизационными существенными многочленами.

Сушествование факторизационных сушественных многочленов у последовательности $c_{-\kappa}, \ldots, c_{0}, \ldots, c_{\kappa}$ доказано в теореме 3.1. Оказывается, что с помошью любых факторизационных сушественных многочленов можно восстановить факторизационные множители $l_{+}(t), r_{+}(t)$ и тем самым построить факторизации $(1),(2)$. Окончательный результат выглядит следуюшим образом.

Теорема 3.2. Пусть а(t) - матрица-функция, аналитическая в области $D_{+}$, непрерывная в области $D_{+} \cup \Gamma$ и обратимая на контуре $\Gamma$. Пусть $\Delta(t)=\Delta_{-}(t) t^{\kappa} \Delta_{+}(t)-$ факторизация Винера-Хопфа определителя $\operatorname{det} a(t)$. Coставим из моментов матрицы-функции $\Delta_{-}^{-1}(t) a(t)$ относительно контура $\Gamma$ последовательность $c_{-\kappa}, \ldots, c_{0}, \ldots, c_{\kappa}$. Тогда эта последовательность регулярна и обладает факторизационньми существенными многочленами. Пусть $\mu_{1}, \ldots, \mu_{2 p}$ - индекси последовательности, а $R_{1}(t), \ldots, R_{p}(t), L_{p+1}(t), \ldots, L_{2 p}(t)-$ любые ее факторизачионние существеннъе многочлены. Тогда левые $\lambda_{1}, \ldots, \lambda_{p}$ и правые $\rho_{1}, \ldots, \rho_{p}$ частные индексы и факторизачионные множители $l_{ \pm}(t)$, $r_{ \pm}(t)$ матричного многочлена а $(t)$ определяются по формулам

$$
\begin{aligned}
\lambda_{j} & =-\mu_{j}, & \rho_{j} & =\mu_{p+j}, \quad j=1, \ldots, p, \\
l_{-}(t) & =t^{\kappa+1} \Delta_{-}(t) d_{l}^{-1}(t) \mathcal{R}^{-1}(t), & l_{+}(t) & =t^{-\kappa-1} \Delta_{-}(t) a(t) \mathcal{R}, \\
r_{-}(t) & =\Delta_{-}(t) \mathcal{L}^{-1}(t), & r_{+}(t) & =\Delta_{-}^{-1}(t) \mathcal{L}(t) a(t),
\end{aligned}
$$

əде

$$
\mathcal{R}(t)=\left(R_{1}(t), \ldots, R_{p}(t)\right), \quad \mathcal{L}(t)=\left(\begin{array}{c}
L_{p+1}(t) \\
\vdots \\
L_{2 p}(t)
\end{array}\right)
$$


ДокаЗАТЕЛьство. Требует доказательства лишь утверждение, что факторы $l_{+}(t)$, $r_{+}(t)$ можно восстановить по любым факторизационным существенным многочленам.

Прежде всего покажем, что если $R_{1}(t), \ldots, R_{p}(t)$ - факторизационные сушественные многочлены и $\widehat{R}_{1}(t), \ldots, \widehat{R}_{p}(t)$ - любые другие факторизационные сушественные многочлены, то

$$
\widehat{R}_{j}(t)=\sum_{i=1}^{p} q_{i j}(t) R_{i}(t), \quad j=1,2, \ldots, p,
$$

где $q_{i j}(t)$ - скалярный многочлен от $t$ степени не выше $\mu_{j}-\mu_{i}$, если $\mu_{j}-\mu_{i} \geqslant 0$, и $q_{i j}(t) \equiv 0$, если $\mu_{j}-\mu_{i}<0$. При $\mu_{j}<\mu_{p+1}$ это сразу следует из структуры ядра матрицы $T_{\mu_{j}+1}$. Пусть $\mu_{j}=\mu_{p}=\mu_{p+1}$. В этом случае $\mu_{p}=\mu_{p+1}=0$. Пусть $\mu_{p}=\cdots=\mu_{p+s}=0<\mu_{p+s+1}$. Если $\widehat{R}_{j}(t) \in \mathcal{N}_{1}^{R}-$ любой другой правый факторизационный существенный многочлен, соответствующий индексу $\mu_{j}=0$, то, разложив его по базису $\mathcal{N}_{1}^{R}$, имеем

$$
\widehat{R}_{j}(t)=\sum_{i=1}^{p} q_{i j}(t) R_{j}(t)+q_{p+1, j} R_{p+1}(t)+\cdots+q_{p+s, j} R_{p+s}(t)
$$

Здесь $q_{i j}(t)$ - многочлен степени не выше $\mu_{j}-\mu_{i}$ для $i=1, \ldots, p$ и $q_{i j}-$ константы для $i=p+1, \ldots, p+s$. Учитывая, что для факторизационных существенных многочленов свободные члены равны нулю, получаем

$$
q_{p+1, j} R_{p+1,0}+\cdots+q_{p+s, j} R_{p+s, 0}=0 .
$$

Однако для факторизационных существенных многочленов обратимая матрица $\Lambda_{R}$ из критерия существенности является блочно-треугольной. Поэтому свободные члены существенных многочленов $R_{p+1}(t), \ldots, R_{2 p}(t)$ должны быть линейно независимы. Значит, равенство (8) возможно, только если $q_{p+1, j}=\cdots=q_{p+s, j}=0$. Таким образом, разложение (7) полностью доказано.

Образуем из новых факторизационных существенных многочленов матрицу-функцию $\widehat{\mathcal{R}}(t)=\left(\widehat{R}_{1}(t), \ldots, \widehat{R}_{p}(t)\right)$. Из разложения $(7)$ следует, что

$$
\widehat{\mathcal{R}}(t)=\mathcal{R}(t) Q(t)
$$

где $Q(t)$ - матричный многочлен, элементы которого удовлетворяют условию $q_{i j}(t) \equiv 0$, если $\mu_{j}-\mu_{i}<0$, и $q_{i j}(t)$ - многочлен от $t$ формальной степени $\mu_{j}-\mu_{i}$, если $\mu_{j}-\mu_{i} \geqslant 0$. Точно так же можно получить представление

$$
\mathcal{R}(t)=\widehat{\mathcal{R}}(t) \widehat{Q}(t),
$$

где $\widehat{Q}(t)$ имеет такую же структуру, что и $Q(t)$. 
В теореме 3.1 было доказано сушествование обратимой матрицы-функции $\mathcal{R}(t)$. Поэтому

$$
Q(t) \widehat{Q}(t)=I_{p},
$$

т.е. матричный многочлен $Q(t)$ обратим. Обозначим

$$
\hat{l}_{-}(t)=t^{\kappa+1} \Delta_{-}(t) d_{l}^{-1}(t) \widehat{\mathcal{R}}^{-1}(t) .
$$

Тогда матрица

$$
\hat{l}_{-}(t)=d_{l}^{-1}(t) Q^{-1}(t) d_{l}(t) l_{-}(t)
$$

удовлетворяет условиям теоремы об общем виде факторизационных множителей левой факторизации [5]. Это означает, что $\hat{l}_{-}(t)$ также является факторизационным множителем. Вторая часть теоремы доказывается аналогично.

Задача факторизации Винера-Хопфа тесно связана со спектральной факторизацией аналитических матриц-функций $a(t)$ (в частности, матричных многочленов), т.е. с задачей выделения делителя $a(t)$, ответственного за часть спектра $a(t)[6,7]$. Эта задача также весьма актуальна в теории дискретных аналогов интегрируемых систем, где важную роль играет факторизация матричных многочленов [8]. Для простоты мы ограничимся выделением делителей $a(t)$, ответственных только за одну точку спектра $a(t)$. В этом случае последовательность составляется из коэффициентов Тейлора матрицы-функции $a(t)$, а сушественные многочлены последовательности тесно связаны с жордановыми цепочками $a(t)$.

Пусть $a(t)$ - матрица-функция, аналитическая в некоторой области $D \subset \mathbb{C}$, и $\operatorname{det} a(t)$ не равен нулю тождественно. Точки, в которых $\operatorname{det} a(t)=0$, назовем собственными значениями $a(t)$, а все остальные точки - регулярными. Пусть $t_{0}$ - собственное значение $a(t)$ алгебраической кратности $\kappa$. Составим последовательность $0, \ldots, 0, c_{0}, \ldots, c_{\kappa}$ из коэффициентов Тейлора $c_{j}=1 / j ! a^{(j)}\left(t_{0}\right)$ в точке $t_{0}$. Здесь $a^{(j)}\left(t_{0}\right)$ - значение $j$-й производной $a(t)$ в точке $t_{0}$. Индексы этой последовательности назовем индексами аналитической матрищы-функции $a(t)$ в точке $t_{0}$. Очевидно, что для регулярных точек и только для них все индексы равны нулю.

Пусть $\mathcal{R}(t), \mathcal{L}(t)$ - матрицы, составленные из факторизационных сушественных многочленов последовательности. Пусть $Г$ - любой простой замкнутый контур, окружающий точку $t_{0}$, такой, что внутри него нет других собственных значений $a(t)$. Легко видеть, что для этого контура $\Delta_{-}(t)=1$ и, следовательно, моменты $\Delta_{-}^{-1}(t) a(t)$ совпадают с коэффициентами Тейлора $a(t)$ в точке $t_{0}$. Применение теоремы 3.2 дает следующее следствие.

СЛЕДСТВИЕ 3.1. Пусть $t_{0}$ - собственное значение аналитической матрииь-функиии а(t) алгебраической кратности к. Тогда $r(t)=\left(t-t_{0}\right)^{\kappa+1} \mathcal{R}^{-1}(t)$ является матричным многочленом от $t$ таким, что $a(t)=b(t) r(t)$. Здесь 
$b(t)=\left(t-t_{0}\right)^{-\kappa-1} a(t) \mathcal{R}(t)$ - аналитическая в $D$ матрица-функиия, причем $t_{0}$ ее регулярная точка.

Аналогично $а(t)=l(t) c(t)$, где $l(t)=\mathcal{L}^{-1}(t) d_{r}(t)$ - матричный многочлен от $t$ и $c(t)=d_{r}^{-1}(t) \mathcal{L}(t) a(t)$ - аналитическая матрица-функиия, для которой $t_{0}-$ регулярная точка.

С помошью предложенного в данной статье метода можно также указать необходимые и достаточные условия существования линейного делителя матрицы-функции $a(t)$, ответственного за нуль ее определителя. При этом не предполагается, что делитель является регулярным, т.е. имеет обратимый старший коэффициент.

СЛЕДСТВИЕ 3.2. Пусть аналитическая в $D$ матрица-функция $а(t)$ имеет в точке $t_{0} \in D$ нуль определителя кратности $\kappa>0$. Тогда, для того чтобы существовал линейный правый (левый) делитель $r(t) \quad(l(t))$ матрицы-функиии $а(t)$ такой, что $\operatorname{det} r(t)=\left(t-t_{0}\right)^{\kappa} \quad\left(\operatorname{det} l(t)=\left(t-t_{0}\right)^{\kappa}\right)$, необходимо и достаточно, чтобъ индексы $\mu_{1}, \ldots, \mu_{p}\left(\mu_{p+1}, \ldots, \mu_{2 p}\right)$ матрицы-функиии $а(t)$ в точке $t_{0}$ бълли равнь -1 или 0 (1 или 0). При этом делитель является регулярным тогда и только тогда, когда $\kappa=p$.

Эти результаты легко переносятся и на общий случай делителя, ответственного за часть спектра $a(t)$.

\section{Список литературы}

[1] В. Е. Захаров, А. Б. Шабат. Функц. анализ и его прилож. 1979. Т. 13. № 3. С. 13-22.

[2] А. Б. Шабат. Дифф. уравнения. 1979. Т. 15. № 10. С. 1824-1834.

[3] В. М. Адуков. Алгебра и анализ. 1992. Т. 4. № 1. С. 54-74.

[4] V. M. Adukov. Linear Algebra and Its Appl. 1998. V. 274. P. 85-124.

[5] И. Ц. Гохберг. УМН. 1964. Т. 19. № 1. С. 71-124.

[6] A. С. Маркус. Введение в спектральную теорию полиномиальных операторных пучков. Кишинев: Штиинца, 1986.

[7] I. Gohberg, P. Lancaster, L. Rodman. Matrix polynomials. N. Y.: Academic Press, 1982.

[8] А. П. Веселов. Функц. анализ и его прилож. 1991. Т. 25. № 2. С. 38-49. 